Check for updates

The BMJ

Cite this as: $B M / 2021 ; 373: n 908$ http://dx.doi.org/10.1136/bmj.n908 Published: 07 April 2021

\title{
Covid-19: One in three has neurological or psychiatric condition diagnosed after covid infection, study finds
}

\section{Elisabeth Mahase}

One in three people who have had covid-19 then have a neurological or psychiatric condition diagnosed within six months of infection-the first such diagnosis in $13 \%$ of patients-a study of US patient records has found. ${ }^{1}$

When compared with people who had flu or other respiratory tract infections over the same period, covid-19 survivors were more likely to have conditions such as anxiety and mood disorders diagnosed, in research that included 236379 patients with covid-19 from the US TriNetX electronic health records network.

"Given the size of the pandemic and the chronicity of many of the diagnoses and their consequences, substantial effects on health and social care systems are likely to occur," said the research team from the University of Oxford, UK. "Our data provide important evidence indicating the scale and nature of services that might be required.

"The findings also highlight the need for enhanced neurological follow-up of patients who were admitted to intensive care or had encephalopathy during their covid-19 illness."

Looking at the severity of covid-19, the researchers found that nearly half of people who had been admitted to intensive care (46\%) had a neurological or psychiatric condition diagnosed within six months (a first diagnosis for $26 \%$ ), and this figure rose to $62 \%$ in patients who had had delirium (encephalopathy) during their covid-19 illness.

\section{Severity of conditions}

The rates of individual conditions also increased with severity of covid-19, the team reported in the Lancet Psychiatry. ${ }^{1}$ While $0.3 \%$ of people who had not required hospital admission had a brain haemorrhage, this rose to $2.7 \%$ among those who had been in intensive care and in $3.6 \%$ of people who had had delirium. Ischaemic stroke incidence rose from $1.3 \%$ after no hospital admission to $6.9 \%$ after intensive care and 9.4\% after delirium. Psychotic disorder incidence increased from $0.9 \%$ (no admission) to $2.8 \%$ (intensive care) to $7 \%$ (delirium).

However, the researchers noted a number of limitations to their study, including that they looked at a maximum of six months post-infection, they did not consider the severity of the subsequent conditions, and they were unable to use baseline rates as a comparison.

As an alternative, the team used other respiratory conditions experienced over the same pandemic period as a comparison. After adjusting for underlying health characteristics, such as age, sex, ethnicity, and existing conditions, they reported a $44 \%$ greater risk of neurological and mental health diagnoses after covid-19 than after flu and a 16\% greater risk after covid-19 than with respiratory tract infections.

Specific neurological diagnoses highlighted by the researchers included ischaemic stroke, intracranial haemorrhage, and dementia.

The paper said, "Consistent with several other reports, the risk of cerebrovascular events (ischaemic stroke and intracranial haemorrhage) was elevated after covid-19, with the incidence of ischaemic stroke rising to almost one in ten (or three in 100 for a first stroke) in patients with encephalopathy. A similarly increased risk of stroke in patients who had covid-19 compared with those who had influenza has been reported."

Looking at dementia, the researchers said that although the estimated incidence was modest overall, $2.66 \%$ of patients with covid-19 aged over 65 and $4.72 \%$ who had had encephalopathy received a first dementia diagnosis within six months of having covid-19.

Masud Husain, study author and professor of neurology and cognitive neuroscience at the University of Oxford, said, "It's difficult to model how many of those people would have just come to us anyway. But if you look at the [dementia] data for the patients who had flu versus those with covid, that's quite a sizeable increase [covid-19 $v$ influenza hazard ratio 2.33]."

Husain added that, beyond raw numbers, the time at which patients present and have a condition diagnosed may be important. "It could be that over the next year we're going to see a lot more patients being referred with dementia, but that group of people might nevertheless have come to us anyway in two years' time," he said.

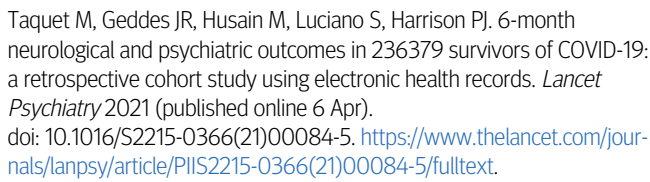

This article is made freely available for use in accordance with BMJ's website terms and conditions for the duration of the covid-19 pandemic or until otherwise determined by BMJ. You may use, download and print the article for any lawful, non-commercial purpose (including text and data mining) provided that all copyright notices and trade marks are retained. 